\title{
Non-spatial impairments affect false positive neglect diagnosis based on cancellation tasks
}

\author{
Hanne Huygelier ${ }^{\mathrm{a}}$, Margaret Jane Moore ${ }^{\mathrm{b}}$, Nele Demeyere ${ }^{\mathrm{b}}$, \& Céline R. Gillebert ${ }^{\mathrm{a}, \mathrm{b}}$ * \\ ${ }^{a}$ Department of Brain and Cognition, KU Leuven, Leuven, Belgium \\ ${ }^{\mathrm{b}}$ Department of Experimental Psychology, University of Oxford, Oxford, United Kingdom \\ *Corresponding author: Céline R. Gillebert, Tiensestraat 102, box 3711, Leuven 3000, Belgium \\ (e-mail: celine.gillebert@ kuleuven.be and telephone: +32 163727 04). ORCID ID: \\ https://orcid.org/0000-0001-6686-7262
}

Word count abstract: 247

Word count manuscript (excluding abstract/figures/tables/references): 4999 


\begin{abstract}
Objective. To diagnose egocentric neglect after stroke, the spatial bias of performance on cancellation tasks is typically compared to a single cut-off. This standard procedure relies on the assumption that the measurement error of cancellation performance does not depend on non-spatial impairments affecting the total number of cancelled targets. Here we assessed the impact of this assumption on false positive diagnoses.
\end{abstract}

Method. We estimated false positives by simulating cancellation data using a binomial model. Performance was summarized by the difference in left and right cancelled targets (R-L) and the Centre of Cancellation $(\mathrm{CoC})$. Diagnosis was based on a fixed cut-off versus cut-offs adjusted for the total number of cancelled targets, and on single test performance versus unanimous or proportional agreement across multiple tests. Finally, we compared the simulation findings to empirical cancellation data acquired from 651 stroke patients.

Results. Using a fixed cut-off, the rate of false positives depended on the total number of cancelled targets, and ranged from 10 to $30 \%$ for R-L scores and from 10 to $90 \%$ for CoC scores. The rate of false positives increased even further when diagnosis was based on proportional agreement across multiple tests. Adjusted cut-offs and unanimous agreement across multiple tests were effective at controlling false positives. For empirical data, fixed versus adjusted cut-offs differ in estimation of neglect prevalence by $13 \%$, and this difference was largest for patients with non-spatial impairments.

Conclusions. Our findings demonstrate the importance of considering non-spatial impairments when diagnosing neglect based on cancellation performance.

Keywords: Perceptual Disorders, Task Performance and Analysis, Psychometrics, Stroke, Clinical Decision Making, Neuropsychology 


\section{Introduction}

Egocentric spatial neglect is characterized by an inability to respond to stimuli presented in the contralesional hemifield (Heilman, Bowers, Valenstein, \& Watson, 1987). Neglect is commonly diagnosed using a test battery including cancellation tasks (Albert, 1973; Azouvi et al., 2006; Donnelly et al., 1999; Rorden \& Karnath, 2010; Vaes et al., 2015; Weintraub \& Mesulam, 1988). In these tasks, patients with egocentric neglect cancel fewer targets on the contralesional than on the ipsilesional side of space, while healthy controls cancel a high number of targets evenly throughout the search array (Dalmaijer, Stigchel, Nijboer, Cornelissen, \& Husain, 2014; Donnelly et al., 1999; Gauthier, Dehaut, \& Joanette, 1989).

Performance on cancellation tasks can be quantified by comparing the number of cancelled targets on the left and right side of space (R-L score) or with the Centre of Cancellation (CoC) which represents the average location of cancelled targets (e.g. Azouvi et al., 2006; Demeyere \& Gillebert, 2019; Demeyere, Riddoch, et al., 2015; Robertson et al., 1994; Rorden \& Karnath, 2010; Vaes et al., 2015). These measures aim to capture the spatial asymmetry typical of neglect, that is the difference in the probability to cancel targets between the left and right side of the cancellation display (Huygelier \& Gillebert, 2018). To establish whether a patient has neglect, the R-L or CoC score is compared to a single impairment threshold, not considering the total number of cancelled targets (e.g. Demeyere et al., 2015; Rorden \& Karnath, 2010). We refer to this type of thresholds as fixed normative cut-offs. Fixed normative cut-offs are based on percentiles of test scores obtained in neurologically healthy individuals or stroke patients without egocentric neglect (e.g. Demeyere, Riddoch, et al., 2015; Rorden \& Karnath, 2010). These percentiles represent the most extreme test scores that can be observed in individuals without neglect and are expected to limit false positive diagnosis. 


\section{Measurement Precision of Cancellation Performance Depends on Non-Spatial Impairments}

Despite the popularity of cancellation tasks, few studies investigated their measurement precision (Bailey, Riddoch, \& Crome, 2004; Machner, Mah, Gorgoraptis, \& Husain, 2012). Measurement precision is important as it affects the certainty with which conclusions can be drawn based on test scores (Crawford \& Garthwaite, 2002; Lord, 1952; Lord, Novick, \& Birnbaum, 1968; Slick, 2006). In classic test theory, it is assumed that the observed test score of a person on a specific moment can be divided in two parts: the true underlying test score ${ }^{1}$ that remains stable across test moments and the measurement error that leads to probabilistic variation of observed test scores across moments (Novick, 1966). Thus, the observed score of an individual is considered an estimate of the true underlying score (Lord, Novick, \& Birnbaum, 1968; Slick, 2006) and measurement precision refers to the extent to which an observed score reflects the true score. When measurement precision is low, the chance is low that we can observe the same score across repeated assessments.

Bailey et al. (2004) and Machner et al. (2012) estimated the measurement error of the total number of cancellations and the R-L score divided by the total number of cancellations by studying the variability in these scores across repeated assessments in neglect patients. The authors observed considerable variability in cancellation scores across repeated testing. However, by estimating the measurement error across all patients, they treated the error as a constant property, reflecting the assumption that each patient's observed score is associated with the same level of error. Measurement error is indeed considered a constant property of scores in classic test theory (Novick, 1966), but this principle may not apply to cancellation scores. That is, in contrast to continuous

\footnotetext{
${ }^{1}$ We deliberately use the term "true underlying test score" and not "true deficit". Whether the true underlying test score represents the true underlying deficit depends on the validity of the task and is an independent issue from the measurement error.
} 
outcome measures, responses on a cancellation task are discrete: a patient either cancels or omits a single target.

Given their discrete nature, cancellation responses are best described by a binomial distribution with the mean equal to $N P$ and variance equal to $N P(1-P)$, where $N$ is the number of targets and $P$ is the probability to cancel a target. The association between the mean and variance of the distribution (Brown, Thomas, \& Patt, 2017; Lord, 1952; Lord et al., 1968; McDonald, 2011) implies that the measurement error of cancellation test scores is variable. Applying the binomial formula to a cancellation task with 50 targets reveals that we can expect, for instance, a variance of 12.5 cancelled targets across repeated tests for a patient with $50 \%$ chance to cancel each target, and a variance of 4.5 cancelled targets for a patient with a 10 or $90 \%$ chance to cancel each target. In other words, the measurement error of the number of cancelled targets is highest when the probability to cancel a target equals $50 \%$ and decreases as the probability approximates 0 or $100 \%$. Binomial variance affects test scores that rely on the number of cancelled targets such as the R-L score in the same way.

This non-constant error variance may be important since stroke patients likely vary in their ability to cancel targets in a non-lateralized way, or in other words in the number of omissions made across the visual field. We will refer to the reduced probability of cancelling targets irrespective of their location in the visual field as non-spatial errors (Huygelier \& Gillebert, 2018). Non-spatial errors can result from a mix of multiple non-spatial impairments, including, among others, impairments in selective and sustained attention (Foldi, Jutagir, Davidoff, \& Gould, 1992) and working memory (Husain et al., 2001). The measurement error of cancellation test scores will be higher in stroke patients with versus without such non-spatial impairments. Consequently, the most extreme R-L and $\mathrm{CoC}$ scores that can be observed in individuals without neglect will depend on non-spatial 
impairments. Therefore, it is unclear whether fixed normative cut-offs can successfully limit the rate of false positive neglect diagnoses as these cut-offs ignore non-spatial errors.

The chance of false positive neglect diagnosis also depends on how multiple test instruments inform the diagnosis. Although it is not uncommon to diagnose neglect on a single cancellation task (e.g. Brink, Verwer, Biesbroek, Visser-Meily, \& Nijboer, 2017; Demeyere \& Gillebert, 2019; Farnè et al., 2004; Nijboer, Kollen, \& Kwakkel, 2013), many researchers and clinicians use multiple tasks (e.g. cancellation, line bisection and figure copying) to diagnose egocentric neglect. There is however no gold-standard approach for reconciling conflicting diagnoses from multiple tests, as diagnostic criteria vary considerably across published studies. Some studies required unanimous diagnostic agreement from two cancellation tasks (e.g. Smania et al., 1998), while others required a certain proportion of agreement across multiple tasks varying from $17 \%$ to $67 \%$ (e.g. Cazzoli et al., 2012; Dalmaijer et al., 2018; McIntosh, Schindler, Birchall, \& Milner, 2005; Plummer, Dunai, \& Morris, 2006; Rengachary, He, Shulman, \& Corbetta, 2011; Rorden \& Karnath, 2010; Urbanski et al., 2010; Verdon, Schwartz, Lovblad, Hauert, \& Vuilleumier, 2010). The impact of these different methods on the rate of false positives has not been investigated yet.

\section{The Present Study}

The purpose of the present study is to provide insights in how non-spatial impairments affect false positive neglect diagnosis. To this end, we simulated cancellation data using a simple probabilistic model (Huygelier \& Gillebert, 2018). We used Monte Carlo simulation, a method to make inferences using random numbers that follow a certain probability distribution (i.e. in our case the binomial distribution) (Beisbart \& Norton, 2012). This method allows full control over the true test score underlying the observed test score, making it valuable for psychometric research (Feinberg 
\& Rubright, 2016) and providing theoretical insights that can aid clinical decision making (Beaujean, 2018).

Using these simulated data, we assessed the impact of fixed cut-offs on false positive diagnosis. We predicted that the measurement precision of R-L scores would be lowest in simulated cases who had $50 \%$ non-spatial errors, which in turn would result in an inflated false-positive neglect diagnosis. For the $\mathrm{CoC}$, we predicted the lowest measurement precision and highest false positive rates for the highest percentages of non-spatial errors, in line with earlier findings (Toraldo et al., 2017). This procedure was compared to a new approach, in which cut-offs were adjusted according to the total cancelled targets. We predicted better control over false positives using adjusted cutoffs compared to fixed cut-offs. Moreover, we assessed the impact of different diagnostic methods on false positives. Neglect was either diagnosed on a single test or on multiple tests. In case of multiple tests, we compared an approach of diagnosing neglect on unanimous versus proportional agreement of test results.

Finally, we aimed to illustrate the real-world impact of our premises using cancellation data acquired from a cohort of 651 stroke patients. In this analysis, we first assessed the occurrence and the distribution of non-spatial errors in our sample of stroke patients. We then assessed the percentage of patients for which fixed normative versus adjusted cut-offs would lead to a different diagnosis. Finally, we assessed whether fixed normative versus adjusted cut-offs differed most in rates of diagnosing neglect for patients who made approximately $50 \%$ non-spatial errors. 


\section{Method}

\section{The Theoretical Impact of Diagnostic Methods on False Positive Rates}

\section{Simulating cancellation data using a binomial model}

To simulate cancellation data, we generated 50 uniformly distributed target locations ranging from -1 (left border of search matrix) to +1 (right border of search matrix). We chose 50 targets, since this approximates the number of targets of many cancellation tasks: the Bells test with 35 targets (Gauthier et al., 1989), line cancellation with 40 targets (Albert, 1973), diamond cancellation with 48 targets (Vaes et al., 2015), OCS cancellation with 50 targets (Demeyere et al., 2015), Star cancellation with 54 targets (Halligan, Cockburn, \& Wilson, 1991) and the letter cancellation with 60 targets (Weintraub, 1985).

Then, cancellation responses for each target were simulated according to a simple binomial model (see Huygelier \& Gillebert, 2018). The model assumes that cancellation responses result from a probabilistic process in which each target has a certain probability to be cancelled. In case of egocentric neglect, the probability underlying cancellation responses on the contralesional side is smaller than the probability underlying cancellation responses on the ipsilesional side of the cancellation page. The difference between these two probabilities is referred to as "spatial asymmetry". For patients with no neglect, these probabilities are equal. The cancelled targets depend on these two probabilities and are randomly generated using a binomial distribution.

Importantly, our model assumes that the probability to cancel targets across the entire display can be smaller than 1, allowing for non-spatial errors to occur. Note that in case of no true spatial asymmetry, non-spatial errors will be directly related to overall cancellation performance. That is, if a patient has a probability of cancelling $50 \%$ of all targets across the cancellation array, then the 
expected total cancelled targets will be equal to $50 \%$. In case of a true spatial asymmetry, the overall cancellation performance can reflect a combination of non-spatial and spatial errors. Using this model, cancellation responses were simulated for patients with no spatial asymmetry and with varying levels of non-spatial errors. The level of non-spatial errors ranged from 0 to 1 in 10 steps with 10,000 simulations for each level, producing a dataset with 110,000 simulated observations.

Finally, the R-L and CoC scores were calculated for each of these simulated cancellation tasks. The R-L score was calculated by subtracting the proportion cancelled targets for the 25 targets located on the left side from that of the 25 targets located on the right side. The CoC was calculated by averaging the location of cancelled targets and subtracting the average location of all targets. Both R-L and CoC scores ranged from -1 to +1 , where negative values indicated more cancelled targets on the left than right side and vice versa.

\section{Estimating fixed and adjusted cut-offs}

The $5^{\text {th }}$ and $95^{\text {th }}$ percentiles of the R-L and CoC scores were estimated based on our simulated dataset. Two types of R-L and CoC cut-offs were calculated; fixed and adjusted cut-offs. To estimate the fixed cut-offs, a subset of simulated data in which at least $80 \%$ targets had been cancelled was chosen as this performance is similar to that of neurologically healthy individuals as reported for instance in Demeyere et al. (2015). Adjusted cut-offs were determined on subsets of simulated data, each with a specific proportion ${ }^{2}$ of cancelled targets ranging from 0 to 1 in 10 steps.

\footnotetext{
${ }^{2}$ Data were divided in subsets on the expected proportion cancelled targets, because all observations that result from the same level of expected performance have the exact same binomial variance and would therefore lead to adjusted cut-offs that represent this binomial variance.
} 
False positives for different diagnostic methods

Fixed and adjusted cut-offs were used to interpret the simulated cancellation test scores. Each of the 110,000 simulated individual performances were classified into one of three categories ${ }^{3}$. For a single test administration, observations with a R-L or CoC score greater than the $95^{\text {th }}$ percentile were classified as left neglect, while observations with a R-L score lower than the $5^{\text {th }}$ percentile were classified as right neglect. Single observations with R-L or CoC scores between the $5^{\text {th }}$ and $95^{\text {th }}$ percentile were classified as no neglect. This classification was performed separately for the fixed and adjusted cut-offs. For the unanimous agreement, the number of administered tests varied from 2 to 3 and neglect was only diagnosed when each of the administered tests consistently indicated neglect for the same side of space (i.e. consistent left-sided or consistent right-sided neglect). For the proportional agreement, we simulated 5 test administrations and varied the minimum number of positive test results required for diagnosis from 1 to 4 . Then, for each classification method we calculated the rate of false positive diagnoses. Note that false positives should remain below $10 \%$ when testing for the presence of left- and right-sided neglect (two-sided testing) when using the $5^{\text {th }}$ and $95^{\text {th }}$ percentiles. That is, as $10 \%$ of the control group without neglect have test scores that exceed these percentiles, you expect $10 \%$ false positive diagnosis.

\section{The Real-World Impact of Fixed Normative versus Adjusted Cut-offs on Neglect Diagnosis}

A consecutive sample of stroke survivors were recruited from the John Radcliffe Hospital (Oxford, UK) between February 2012 and September 2018 in compliance with the regulations of the National Research Ethics Service (11/WM/0299 and 14/LO/0648) and Helsinki declaration.

\footnotetext{
${ }^{3}$ Note that by generating 110,000 observations the expected values for the cut-offs are obtained. These expected values will not vary across repetitions of these simulations.
} 
Patients were included if they were able to remain alert for 20 minutes and were able to provide informed consent. Participant characteristics are reported in Table 1.

\section{[INSERT TABLE 1 HERE]}

The OCS cancellation task is a search matrix of 150 heart drawings pseudo-randomly scattered across a landscape orientation A4 page. Two thirds of these drawings have left or right gaps (distractors) and the remaining third are complete drawings (targets). These drawings are arranged according to a grid pattern ensuring that there are an equal number of targets and distractors across different areas of the page. Patients were asked to cross out all complete hearts. Patients were given two practice trials before proceeding to the full task. Patients who were unable to hold a pen responded by pointing to each stimulus which was then immediately marked by the examiner. Each patient was allowed three minutes to complete the task. Performance was summarized by subtracting the number of cancelled targets on the right side from the left side of this array. This R-L score ${ }^{4}$ ranged from -20 to 20 . Demeyere et al. (2015) reported fixed normative cut-offs $\left(5^{\text {th }}\right.$ and $95^{\text {th }}$ percentiles) of 3 and -2 .

Using these empirical data, we evaluated the implications of our simulations. First, we determined the prevalence of non-spatial errors in stroke patients without statistically significant neglect according to the fixed normative cut-offs. For this purpose, we calculated the proportions of patients obtaining a total performance of either 0-10, 11-20, 21-30, 31-40 and 41-50 cancelled targets with a R-L score $\geq-2$ and $\leq 3$. Second, we assessed whether fixed normative versus adjusted cut-offs lead to different diagnostic decisions. To this end, the proportion of patients classified as

\footnotetext{
${ }^{4}$ These analyses focus on the R-L score, because the data did not contain information of each single target location which impeded the computation of CoC scores.
} 
having statistically significant neglect based on the fixed normative cut-offs (i.e. 3 and -2) and our adjusted cut-offs was calculated. For the adjusted cut-offs, stroke patients were categorized according to their total performance in different groups: 0-10, 11-20, 21-30, 31-40, 41-50 cancelled targets. Third, we assessed whether diagnostic decisions based on these two cut-offs differed most for patients who cancelled around 50\% (range: 40 to $60 \%$ targets), for whom we expected the highest false positive rates.

\section{Results}

\section{Fixed and Adjusted Cut-offs}

First, fixed cut-offs were calculated based on the simulated dataset. In simulated cases where at least $80 \%$ of targets were cancelled, R-L scores more extreme than $+/-.12$ represented statistically significant neglect impairment at the $10 \%$ level (Table 2). These cut-offs align well with fixed normative cut-offs with Demeyere et al. (2015) reporting cut-offs of -.10 and .15 and Robertson et al. (1994) reporting +/- .10. CoC scores more extreme than +/- .05 represented statistically significant neglect impairment at the $10 \%$ level based on our simulated data. These CoC cut-offs align well with the normative cut-offs reported by Rorden and Karnath (2010) of +/- .08.

Next, adjusted cut-offs were calculated for each performance level. Adjusted R-L cut-offs ranged from $+/-.24$ in cases where $50 \%$ of targets were expected to be cancelled to $+/-.12$ in cases where $90 \%$ or $10 \%$ of targets were expected to be cancelled (Table 2). Adjusted CoC cut-offs ranged from +/- .51 in cases where $10 \%$ of targets were expected to be cancelled to +/- .05 in cases where $90 \%$ of targets were expected to be cancelled. These adjusted cut-offs illustrate that extreme R-L scores were most likely when the probability to cancel targets was between 40 and $60 \%$. Extreme CoC scores were most likely when the probability to cancel targets was equal to $10 \%$. 
[INSERT TABLE 2 HERE]

\section{False Positive Rates for Different Diagnostic Methods}

\section{A single test administration}

The rate of false positives was calculated based on fixed and adjusted cut-offs for a single test administration (Figure 1A). When R-L scores and fixed cut-offs were used to diagnose neglect, the rate of false positives increased as the non-spatial errors approximated 40-60\%. False positives reached a maximum of $30 \%$ when the non-spatial errors equaled $50 \%$. False positives exceeded the $10 \%$ threshold for non-spatial errors ranging from 20 to $80 \%$. However, when using adjusted cut-offs, false positives remained below the $10 \%$ threshold for non-spatial errors between 20 to $80 \%$. When CoC scores and fixed cut-offs were used to diagnose neglect, false positives increased as the non-spatial errors increased. The false positives exceeded the $10 \%$ threshold for all nonspatial errors larger than $10 \%$ and reached $90 \%$ for the highest level of non-spatial errors. The adjusted CoC cut-offs produced better control over false positives as they remained equal to or below the $10 \%$ threshold for non-spatial errors from 20 to $80 \%$.

\section{[INSERT FIGURE 1 HERE]}

\section{Multiple tests - unanimous agreement}

Next, the false positive rate was calculated in simulated cases where multiple consistent test results were required to diagnose neglect. In simulated cases where unanimous agreement across multiple tests was required for diagnosis, false positives decreased as the number of tests increased both when using fixed and adjusted cut-offs (Figure 1B and Figure 1C). When using fixed cut-offs, RL false positives dropped below the $10 \%$ level when two consistent positive test results were 
required (Figure 1B). CoC false positives dropped below the $10 \%$ threshold when three consistent positive test results were required (Figure 1C).

\section{Multiple tests - proportional agreement}

Finally, the rate of false positives was calculated for simulated cases in which positive test results from only a proportion of administered tests was required to diagnose neglect. In simulated cases where $1 / 5$ positive test results were required for diagnosis, false positives exceeded the $10 \%$ threshold for almost all cases of non-spatial errors when using fixed cut-offs (Figure 2A) and adjusted cut-offs (Figure 2B) for both R-L and CoC scores. More specifically, for fixed cut-offs false positives ranged from 40 to $80 \%$ for R-L scores and from 40 to $100 \%$ for CoC scores for nonspatial errors ranging from 10 to $90 \%$. Thus, false positives exceeded the expected $10 \%$ rate by a fourfold to a nine-fold. When fixed cut-offs were used, false positives fell below the $10 \%$ threshold for R-L scores when $3 / 5$ positive tests were required, but remained above this threshold for $\mathrm{CoC}$ scores even when 4/5 positive tests were required (Figure 2A). Alternatively, when adjusted cutoffs were used, false positives dropped to the $10 \%$ level for R-L and CoC cut-offs when at least 2/5 positive results were required to diagnose neglect (Figure $2 \mathrm{~B}$ ).

\section{[INSERT FIGURE 2 HERE]}

\section{The Real-World Impact of Fixed Normative versus Adjusted Cut-offs on Neglect Diagnosis}

First, we estimated the prevalence of non-spatial errors in stroke patients without spatial asymmetry in cancellation performance. These patients were identified by comparing cancellation responses to the fixed normative cut-offs published by Demeyere et al. (2015). The results showed that $61 \%$ of the stroke patients did not obtain a R-L score indicative of egocentric neglect. Figure 3A illustrates that a considerable number of these patients cancelled a low number of targets. More 
specifically, $7 \%$ of the patients with normal R-L scores cancelled between 0 to 10 targets, $6 \%$ between 11 to 20 targets, $8 \%$ between 21 to 30 targets, $18 \%$ between 31 and 40 targets and $61 \%$ cancelled more than 40 targets. Thus, $32 \%$ of the patients who were not diagnosed with neglect according to fixed normative cut-offs (i.e. no spatial asymmetry in cancellation performance) cancelled between 20 to $80 \%$ of all targets. It is likely that their cancellation performance was affected by non-spatial impairments as they did not show a large difference in cancelled targets between both sides of the visual field, but still failed to cancel many targets. This group of patients represents $19 \%$ of our entire stroke cohort. These results suggest that the inflated false positive rates in our simulations that applied to simulated cases with non-spatial errors from 20 to $80 \%$ and without spatial asymmetries could apply to $19 \%$ of all stroke patients ${ }^{5}$.

Patient cancellation responses were also compared to the fixed normative and the new adjusted cutoffs. When compared to fixed normative cut-offs, $39 \%$ of the 651 patients exhibited statistically significant egocentric neglect. However, when compared to adjusted cut-offs, only $26 \%$ of patients demonstrated significant egocentric neglect. Of the $39 \%$ patients that were considered as neglect patients using the fixed normative cut-offs, $33 \%$ were not diagnosed with neglect according to the adjusted cut-offs. All patients who were diagnosed as neglect patients using the adjusted cut-offs were also diagnosed as neglect patients using the fixed normative cut-offs. The discrepancy between the number of patients diagnosed according to fixed normative versus adjusted cut-offs was highest for patients who cancelled between 40 to $60 \%$ of targets (Figure 3B).

[INSERT FIGURE 3 HERE]

\footnotetext{
5 This may represent an underestimation of the percentage of patients showing no neglect and only non-spatial impairments, as the fixed normative cut-offs can produce false positive diagnosis and we only estimated the prevalence of non-spatial impairments on those patients who were not diagnosed as neglect patients using these liberal cut-offs.
} 


\section{Discussion}

While fixed normative cut-offs of cancellation test scores are frequently used for diagnosing egocentric neglect, our study shows that they do not adequately consider the discrete nature of cancellation responses. Discrete responses are best modeled by the binomial distribution of which the variance is not constant. This non-constant error variance implies that more extreme R-L scores are more likely to occur when the probability to make responses equals 50\% across the cancellation task. By simulating data using a binomial model, we showed that extreme R-L scores were indeed most probable when $50 \%$ of targets were expected to be cancelled. In contrast, extreme CoC scores were more likely to occur when fewer targets were expected to be cancelled. This pattern of $\mathrm{CoC}$ error variance can be explained by the fact that the $\mathrm{CoC}$ relies on averaging the location of cancelled targets and the statistical average is less precise when it is based on a small sample (i.e. number of cancelled targets).

Our findings on the inflated false positive rate for the $\mathrm{CoC}$ were consistent with an earlier study using a logistic model of spatial neglect (Toraldo et al., 2017). Toraldo et al. (2017) predicted inflated false positive rates as high as $97 \%$ when the number of cancelled targets decreases to a minimum and provided a standardized $\mathrm{CoC}$ score to correct for this inflation of false positives associated to non-spatial impairments. Our study adds onto these earlier findings by revealing the impact of multiple tests on false positives, by providing a direct comparison of false positive rates for the $\mathrm{CoC}$ and $\mathrm{R}-\mathrm{L}$ scores and by showing the impact of the non-constant error variance on realworld neglect diagnosis. 


\section{Patients with Non-spatial Impairments More Likely Misdiagnosed as Neglect Patients}

Our simulations showed that false positives depended on non-spatial errors when diagnosis was based on fixed cut-offs. This suggests that fixed cut-offs lead to more liberal diagnostic decisions for certain patients just because those patients cancel fewer targets overall. In other words, our results suggest that patients with non-spatial impairments are more likely misdiagnosed as neglect patients. Real-world data of a large stroke cohort showed that a considerable number of patients likely have non-spatial impairments. Specifically, 32\% of patients without neglect showed signs of non-spatial impairments in our stroke cohort. Moreover, we showed that fixed normative versus adjusted cut-offs led to a difference in the estimated prevalence of neglect of $13 \%$ These findings suggest that inflated false positives associated with non-spatial impairments have impact on the diagnostic specificity for a considerable number of stroke patients. As such, our analyses showed that accurate theory about the measurement precision of cancellation scores has implications for clinical practice. However, since the true underlying test score of patients are not known, further research is needed to determine the best method to balance false positive and false negative diagnosis.

\section{Adjusted Cut-offs and Unanimous Test Results Control False Positives}

The results of this study provide two concrete ways to limit false positives to the $10 \%$ threshold $^{6}$. First, adjusted cut-offs produced a stable rate of false positives that did not depend on the nonspatial errors. Thus, applying adjusted cut-offs leads to diagnostic decisions that are equally liberal for all patients, independent of whether these patients have non-spatial impairments. Second, requiring unanimous positive test results drastically reduced the rate of false positives, even for

\footnotetext{
${ }^{6}$ Our results can also be extrapolated to other levels of false positives. The same methods can be used to limit false positives to each threshold that a researcher or clinician aims to achieve by using different percentiles.
} 
fixed-cut-offs. For the R-L fixed cut-offs, requiring unanimous agreement of two tests effectively reduced false positives and for $\mathrm{CoC}$ fixed cut-offs unanimous agreement of three tests effectively reduced false positives. Some may argue that the ease of interpreting test scores using a fixed cutoff outweighs the benefit of reducing false positives. To illustrate the ease of using adjusted cutoffs, we added an explanation and simulated adjusted cut-offs that can be used to interpret R-L scores of the OCS cancellation task in Supplementary Materials 1.

\section{Multiple Tests Inflate False Positives}

Many researchers and clinicians already integrate data of multiple tests to diagnose neglect. However, rather than requiring unanimous agreement of multiple tests, it is not uncommon to use a single positive test result out of multiple tests as the criterion to diagnose neglect (e.g. Verdon et al., 2010; McIntosh et al., 2005; Rengachary, He, Shulman, \& Corbetta, 2011). The results of our simulations reveal that this method dramatically increased false positives when both fixed and adjusted cut-offs were used. This result is not surprising given that the chance that at least one test result will be a false positive increases as the number of tests administered increases (Farcomeni, 2008; Nichols \& Hayasaka, 2003). The effect of multiple tests on false positives depends on the extent to which each test is independent. Since performance on different tasks used to diagnose neglect are typically correlated, ranging from correlations of .32 to .97 (Azouvi et al., 2003; Bailey et al., 2004; Rorden \& Karnath, 2010; Sperber \& Karnath, 2016) one might be inclined to expect that the impact of multiple tests on false positives is less pronounced than what our simulations suggest. Here it is important to consider that correlations of performance on neglect tasks will be lower in cases without a true spatial deficit due to the fact that the variance in observed scores of those cases mostly reflects measurement error rather than true differences. Thus, for cases who do not have a true spatial deficit, multiple tests act as independent tests and inflate false positives. 


\section{Reducing False Positives Does Not Necessarily Improve Diagnostic Accuracy}

Throughout this investigation, we have focused on false positive diagnosis. However, accurate diagnosis requires a minimal rate of false positives and false negatives. Depending on the specific goal of the diagnosis, one can choose to give more weight to either avoid false positives or false negatives. For instance, if the aim is to screen patients for neglect, you can choose to use a liberal decision criterion that reduces false negatives rather than false positives. Alternatively, if you aim to test the effect of a new rehabilitation approach you may wish to be more conservative. Indeed, some authors acknowledge that using a criterion of a single positive test result out of multiple tests represents a liberal decision strategy aimed at minimizing false negatives (e.g. McIntosh et al., 2005). Here, we merely aimed to provide insights in how liberal the currently used methods are. Our results revealed that the chance of false positives is not below the $10 \%$ level suggested by the $5^{\text {th }}$ and $95^{\text {th }}$ percentiles, but rather that the chance of false positives often exceeds the $10 \%$ level. More importantly, we showed that the chance of false positives depended on non-spatial errors. These properties of the diagnostic decisions have previously received little attention and could not be adequately considered in clinical decision making.

\section{Clinical Decision Making: More Complicated than Comparing Scores to Cut-offs}

Moreover, the current study focused on a simplified representation of clinical decision making. In clinical practice, neglect diagnosis is informed by test scores, but clinicians typically integrate behavioral observations with test scores. For instance, the search strategy of the patient can add valuable information to interpret cancellation scores. Thus, our results do not necessarily imply that false positive neglect diagnosis are prevalent in clinical practice. Our results do imply that certain diagnostic methods can dramatically increase false positives above the expected $10 \%$ 
threshold and since these methods are used in published studies it is likely that researchers and clinicians were unaware of this.

\section{Conclusions}

To conclude, when you want to control false positive neglect diagnoses, we recommend to base diagnosis on adjusted cut-offs that account for the total performance. Alternatively, if you want to use fixed cut-offs, we recommend to use the R-L score and a criterion of two unanimous test results or the $\mathrm{CoC}$ score and a criterion of three unanimous test results.

\section{Acknowledgements}

The researchers declare no conflicts of interest. This work has been funded by the Research Foundation Flanders (FWO) with a grant awarded to HH (1711717N) and to CRG (G072517N). The patient data were collected as part of an NIHR program development grant (RP-DG-061010046) and NIHR Clinical Research Facility, supported by the National Institute for Health Research Clinical Research Network. ND and MJM are supported by the Stroke Association (TSA LECT 2015/02 and SA PGF 18\100031) 


\section{References}

Albert, M. L. (1973). A simple test of visual neglect. Neurology, 23(6), 658-664. https://doi.org/10.1212/WNL.23.6.658

Azouvi, P., Bartolomeo, P., Beis, J.-M., Perennou, D., Pradat-Diehl, P., \& Rousseaux, M. (2006).

A battery of tests for the quantitative assessment of unilateral neglect. Restorative Neurology and Neuroscience, 24(4-6), 273-285.

Azouvi, P., Olivier, S., de Montety, G., Samuel, C., Louis-Dreyfus, A., \& Tesio, L. (2003). Behavioral assessment of unilateral neglect: Study of the psychometric properties of the Catherine Bergego Scale. Archives of Physical Medicine and Rehabilitation, 84(1), 51-57. https://doi.org/10.1053/apmr.2003.50062

Bailey, M. J., Riddoch, M. J., \& Crome, P. (2004). Test-retest stability of three tests for unilateral visual neglect in patients with stroke: Star Cancellation, Line Bisection, and the Baking Tray Task. Neuropsychological Rehabilitation, 14(4), 403-419. https://doi.org/10.1080/09602010343000282

Beaujean, A. A. (2018). Simulating Data for Clinical Research: A Tutorial. Journal of Psychoeducational Assessment, 36(1), 7-20. https://doi.org/10.1177/0734282917690302

Brink, A. F. T., Verwer, J. H., Biesbroek, J. M., Visser-Meily, J. M. A., \& Nijboer, T. C. W. (2017). Differences between left- and right-sided neglect revisited: A large cohort study across multiple domains. Journal of Clinical and Experimental Neuropsychology, 39(7), 707-723. https://doi.org/10.1080/13803395.2016.1262333 
Brown, G. G., Thomas, M. L., \& Patt, V. (2017). Parametric model measurement: Reframing traditional measurement ideas in neuropsychological practice and research. The Clinical Neuropsychologist, 31(6-7), 1047-1072. https://doi.org/10.1080/13854046.2017.1334829

Cazzoli, D., Müri, R. M., Schumacher, R., von Arx, S., Chaves, S., Gutbrod, K., ... Nyffeler, T. (2012). Theta burst stimulation reduces disability during the activities of daily living in spatial neglect. Brain: A Journal of Neurology, 135(Pt 11), 3426-3439. https://doi.org/10.1093/brain/aws182

Crawford, J. R., \& Garthwaite, P. H. (2002). Investigation of the single case in neuropsychology: Confidence limits on the abnormality of test scores and test score differences. Neuropsychologia, 40(8), 1196-1208. https://doi.org/10.1016/S0028-3932(01)00224-X

Dalmaijer, E. S., Stigchel, S. V. der, Nijboer, T. C. W., Cornelissen, T. H. W., \& Husain, M. (2014). CancellationTools: All-in-one software for administration and analysis of cancellation tasks. Behavior Research Methods, 47(4), 1065-1075. https://doi.org/10.3758/s13428-014$0522-7$

Demeyere, N., \& Gillebert, C. R. (2019). Ego- and allocentric visuospatial neglect: Dissociations, prevalence, and laterality in acute stroke. Neuropsychology, 33(4), 490-498. https://doi.org/10.1037/neu0000527

Demeyere, N., Riddoch, M. J., Slavkova, E. D., Bickerton, W.-L., \& Humphreys, G. W. (2015). The Oxford Cognitive Screen (OCS): Validation of a stroke-specific short cognitive screening tool. Psychological Assessment, 27(3), 883-894. https://doi.org/10.1037/pas0000082 
Donnelly, N., Guest, R., Fairhurst, M., Potter, J., Deighton, A., \& Patel, M. (1999). Developing algorithms to enhance the sensitivity of cancellation tests of visuospatial neglect. Behavior Research Methods, Instruments, \& Computers: A Journal of the Psychonomic Society, Inc, 31(4), 668-673.

Farcomeni, A. (2008). A review of modern multiple hypothesis testing, with particular attention to the false discovery proportion. Statistical Methods in Medical Research, 17(4), 347-388. https://doi.org/10.1177/0962280206079046

Farnè, A., Buxbaum, L. J., Ferraro, M., Frassinetti, F., Whyte, J., Veramonti, T., ... Làdavas, E. (2004). Patterns of spontaneous recovery of neglect and associated disorders in acute right brain-damaged patients. Journal of Neurology, Neurosurgery \& Psychiatry, 75(10), 14011410. https://doi.org/10.1136/jnnp.2002.003095

Feinberg, R. A., \& Rubright, J. D. (2016). Conducting Simulation Studies in Psychometrics. Educational Measurement: Issues and Practice, 35(2), 36-49. https://doi.org/10.1111/emip.12111

Foldi, N. S., Jutagir, R., Davidoff, D., \& Gould, T. (1992). Selective Attention Skills in Alzheimer's Disease: Performance on Graded Cancellation Tests Varying in Density and Complexity. Journal of Gerontology, 47(3), P146-P153. https://doi.org/10.1093/geronj/47.3.P146

Gauthier, L., Dehaut, F., \& Joanette, Y. (1989). The bells test: A quantitative and qualitative test for visual neglect. International Journal of Clinical Neuropsychology.

Halligan, P. W., Cockburn, J., \& Wilson, B. A. (1991). The behavioural assessment of visual neglect. Neuropsychological Rehabilitation, $\quad$ 5-32. https://doi.org/10.1080/09602019108401377 
Heilman, K. M., Bowers, D., Valenstein, E., \& Watson, R. T. (1987). Hemispace and hemispatial neglect. In Neurophysiological and Neuropsychological Aspects of Spatial Neglect: Vol. 45. Advances in Psychology (Vol. 45, pp. 115-150). Retrieved from http://dx.doi.org/10.1016/S0166-4115(08)61711-2

Husain, M., Mannan, S., Hodgson, T., Wojciulik, E., Driver, J., \& Kennard, C. (2001). Impaired spatial working memory across saccades contributes to abnormal search in parietal neglect. Brain, 124(5), 941-952. https://doi.org/10.1093/brain/124.5.941

Huygelier, H., \& Gillebert, C. R. (2018). Quantifying egocentric spatial neglect with cancellation tasks: A theoretical validation. Journal of Neuropsychology, $0(0)$. https://doi.org/10.1111/jnp.12177

Lord, F. (1952). A theory of test scores. Psychometric Monographs, 7, x, 84-x, 84.

Lord, F. M., Novick, M. R., \& Birnbaum, A. (1968). Statistical theories of mental test scores. Oxford, England: Addison-Wesley.

Machner, B., Mah, Y.-H., Gorgoraptis, N., \& Husain, M. (2012). How reliable is repeated testing for hemispatial neglect? Implications for clinical follow-up and treatment trials. J Neurol Neurosurg Psychiatry, 83(10), 1032-1034. https://doi.org/10.1136/jnnp-2012-303296

McDonald, R. P. (2011). Test Theory: A Unified Treatment. New York, NY: Routledge Taylor \& Francis Group.

McIntosh, R. D., Schindler, I., Birchall, D., \& Milner, A. D. (2005). Weights and measures: A new look at bisection behaviour in neglect. Cognitive Brain Research, 25(3), 833-850. https://doi.org/10.1016/j.cogbrainres.2005.09.008 
Na, D. L., Adair, J. C., Choi, S. H., Seo, D. W., Kang, Y., \& Heilman, K. M. (2000). Ipsilesional Versus Contralesional Neglect Depends on Attentional Demands. Cortex, 36(4), 455-467. https://doi.org/10.1016/S0010-9452(08)70532-X

Nichols, T., \& Hayasaka, S. (2003). Controlling the familywise error rate in functional neuroimaging: A comparative review. Statistical Methods in Medical Research, 12(5), 419-446. https://doi.org/10.1191/0962280203sm341ra

Nijboer, T. C., Kollen, B. J., \& Kwakkel, G. (2013). Time course of visuospatial neglect early after stroke: A longitudinal cohort study. Cortex, 49(8), 2021-2027.

Novick, M. R. (1966). The axioms and principal results of classical test theory. Journal of Mathematical Psychology, 3(1), 1-18. https://doi.org/10.1016/0022-2496(66)90002-2

Plummer, P., Morris, M. E., \& Dunai, J. (2003). Assessment of Unilateral Neglect. Physical Therapy, 83(8), 732-740.

Robertson, I. H., Halligan, P. W., Bergego, C., Hömberg, V., Pizzamiglio, L., Weber, E., \& Wilson, B. A. (1994). Right Neglect Following Right Hemisphere Damage? Cortex, 30(2), 199213. https://doi.org/10.1016/S0010-9452(13)80193-1

Rorden, C., \& Karnath, H.-O. (2010). A simple measure of neglect severity. Neuropsychologia, 48(9), 2758-2763. https://doi.org/10.1016/j.neuropsychologia.2010.04.018

Sacchetti, D. L., Goedert, K. M., Foundas, A. L., Barrett, A. M., \& Link to external site, this link will open in a new window. (2015). Ipsilesional neglect: Behavioral and anatomical correlates. Neuropsychology, 29(2), 183-190. http://dx.doi.org/10.1037/neu0000122 
Slick, D. J. (2006). Psychometrics in neuropsychological assessment. In E. Strauss, E. M. Sherman, \& O. Spreen (Eds.), A compendium of neuropsychological tests: Administration, norms and commentary (pp. 3-43). New York, NY: Oxford University Press.

Sperber, C., \& Karnath, H.-O. (2016). Diagnostic validity of line bisection in the acute phase of stroke. $\quad$ Neuropsychologia, $\quad$ 200-204. https://doi.org/10.1016/j.neuropsychologia.2016.01.026

Toraldo, A., Romaniello, C., \& Sommaruga, P. (2017). Measuring and diagnosing unilateral neglect: A standardized statistical procedure. The Clinical Neuropsychologist, 31(6-7), 1248-1267. https://doi.org/10.1080/13854046.2017.1349181

Vaes, N., Lafosse, C., Nys, G., Schevernels, H., Dereymaeker, L., Oostra, K., ... Vingerhoets, G. (2015). Capturing peripersonal spatial neglect: An electronic method to quantify visuospatial processes. Behavior Research Methods, 47(1), 27-44. https://doi.org/10.3758/s13428-014-0448-0

Weintraub, S. (1985). Mental state assessment of young and elderly adults in behavioral neurology. Principles of Behavioral Neurology., 71-123.

Weintraub, S., \& Mesulam, M. M. (1988). Visual hemispatial inattention: Stimulus parameters and exploratory strategies. Journal of Neurology, Neurosurgery, and Psychiatry, 51(12), 14811488. 
Table 1. Demographic and stroke characteristics of stroke sample.

\begin{tabular}{llllll}
\hline \hline Variable & $\mathrm{N}^{\mathrm{a}}$ & $\mathrm{M}$ & $\mathrm{SD}$ & $\mathrm{Min}$ & Max \\
\hline Age & 605 & 74 & 14 & 18 & 97 \\
Years of education & 375 & 12 & 3 & 0 & 23 \\
Handedness & $323 / 25 / 5$ & & & & \\
(right/left/ambidextrous) & & & & & \\
Gender & $291 / 358$ & & & \\
(female/male) & & & & \\
Stroke etiology & $292 / 74 / 17$ & & & \\
(ischemia, hemorrhage, transient ischemic & & & & \\
attack) & & & & \\
Lesion side & $268 / 237 / 22$ & & & \\
(right/left/bilateral) & & & & & \\
Stroke to test interval (days) & 284 & & & & \\
\hline \hline
\end{tabular}

Note. ${ }^{a}$ missing numbers represent patients whose stroke characteristics were unreported in their medical notes. 
Table 2. Fixed and adjusted cut-offs for the two measures of cancellation performance

\begin{tabular}{|c|c|c|c|c|}
\hline \multirow[b]{4}{*}{$\mathrm{P}^{*} \geq .80$} & \multicolumn{2}{|c|}{$\underline{\text { CoC }}$} & \multicolumn{2}{|c|}{$\underline{\mathbf{R}-\mathbf{L}}$} \\
\hline & $\underline{5^{\text {th }} \mathbf{P c}}$ & $9^{\text {th }} \mathbf{P c}$ & $\underline{5^{\text {th }} \mathbf{P c}}$ & $\underline{95}^{\text {th }} \mathrm{Pc}$ \\
\hline & \multicolumn{4}{|c|}{$\underline{\text { Fixed cut-offs }}$} \\
\hline & -.05 & .05 & -.12 & .12 \\
\hline & \multicolumn{4}{|c|}{$\underline{\text { Adjusted cut-offs }}$} \\
\hline $\mathrm{P}=.00$ & .00 & .00 & .00 & .00 \\
\hline$P=.10$ & -.51 & .51 & -.12 & .12 \\
\hline $\mathrm{P}=.20$ & -.33 & .33 & -.20 & .20 \\
\hline $\mathrm{P}=.30$ & -.24 & .24 & -.20 & .20 \\
\hline $\mathrm{P}=.40$ & -.19 & .19 & -.24 & .24 \\
\hline $\mathrm{P}=.50$ & -.15 & .15 & -.24 & .24 \\
\hline$P=.60$ & -.12 & .12 & -.24 & .24 \\
\hline$P=.70$ & -.10 & .10 & -.20 & .20 \\
\hline $\mathrm{P}=.80$ & -.08 & .08 & -.20 & .20 \\
\hline $\mathrm{P}=.90$ & -.05 & .05 & -.12 & .12 \\
\hline $\mathrm{P}=1.00$ & .00 & .00 & .00 & .00 \\
\hline
\end{tabular}

Table Note. $\mathrm{P}=$ expected total performance. $\mathrm{P}^{*}=$ observed total performance. $\mathrm{CoC}=$ the standardized average location of all cancelled targets, $\mathrm{R}-\mathrm{L}=$ the difference between the proportion of cancelled targets on the left versus right visual field. Pc $=$ percentile. 
Figure 1. The simulated false positives as a function of the expected non-spatial errors for the $\mathrm{CoC}$ and R-L score for fixed cut-offs and adjusted cut-offs and for basing the diagnosis on a single test result (A) or on unanimous positive tests results based on two tests (B) and three tests (C). The dashed grey line represents the maximum expected false positive rate based on the $5^{\text {th }}$ and $95^{\text {th }}$ percentiles $(10 \%)$. The $\mathrm{CoC}$ is the standardized average location of all cancelled targets. The R-L is the difference between the proportion of hits on the left and right side of the cancellation array. 
Figure 2. The simulated false positives as a function of the expected non-spatial errors for the CoC and R-L score for fixed cut-offs (A) and adjusted cut-offs (B) and for basing the diagnosis on a minimum of one, two, three, four positive tests out of five administered tests. The dashed grey line represents the maximum expected false positive rate based on the $5^{\text {th }}$ and $95^{\text {th }}$ percentiles $(10 \%)$. The $\mathrm{CoC}$ is the standardized average location of all cancelled targets. The R-L is the difference between the proportion of hits on the left and right side of the cancellation array. 
Figure 3. The relation between the total performance on the hearts cancellation task and R-L scores. The relation of R-L scores and total performance on the hearts cancellation task of 655 stroke patients (A). The blue diamond shapes represent data of the stroke patients. The grey dots represent simulated data based on the binomial model. The black dashed line represents the fixed cut-offs 2 and 3. The red lines represent adjusted cut-offs based on the binomial model. The proportion of patients classified as a neglect patient according to the fixed cut-offs and not according to the adjusted cut-offs is visualized as a function of the total performance on the hearts cancellation task (B). 
Huygelier-Spatial Neglect Diagnosis

A

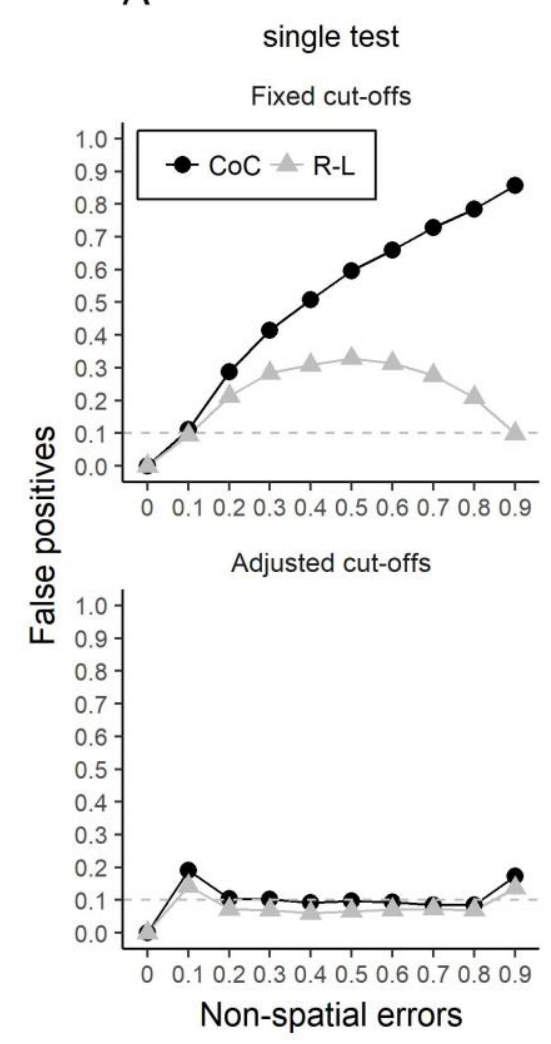

B

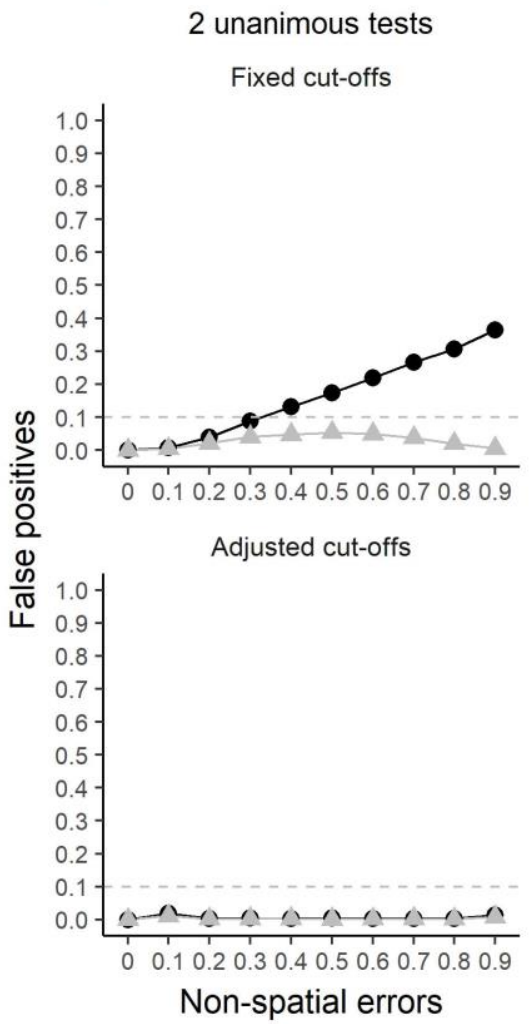

C

3 unanimous tests

Fixed cut-offs

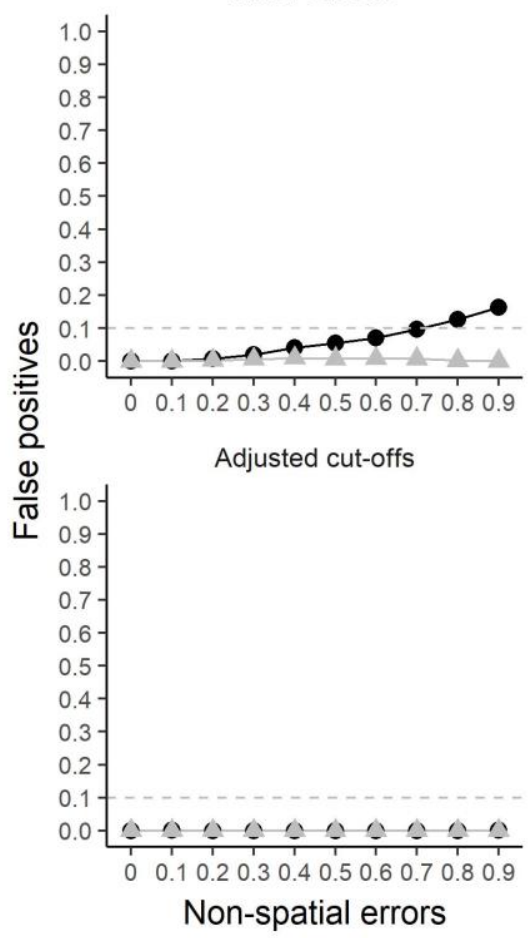

Figure 1. 
Huygelier-Spatial Neglect Diagnosis

A

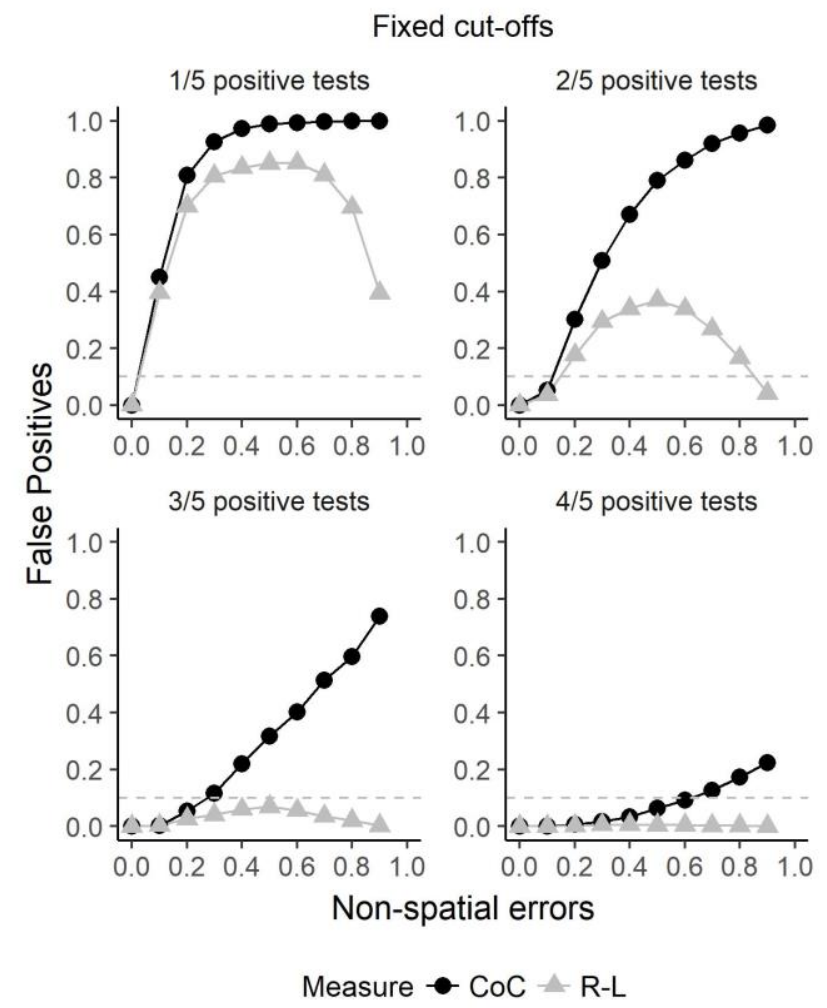

B

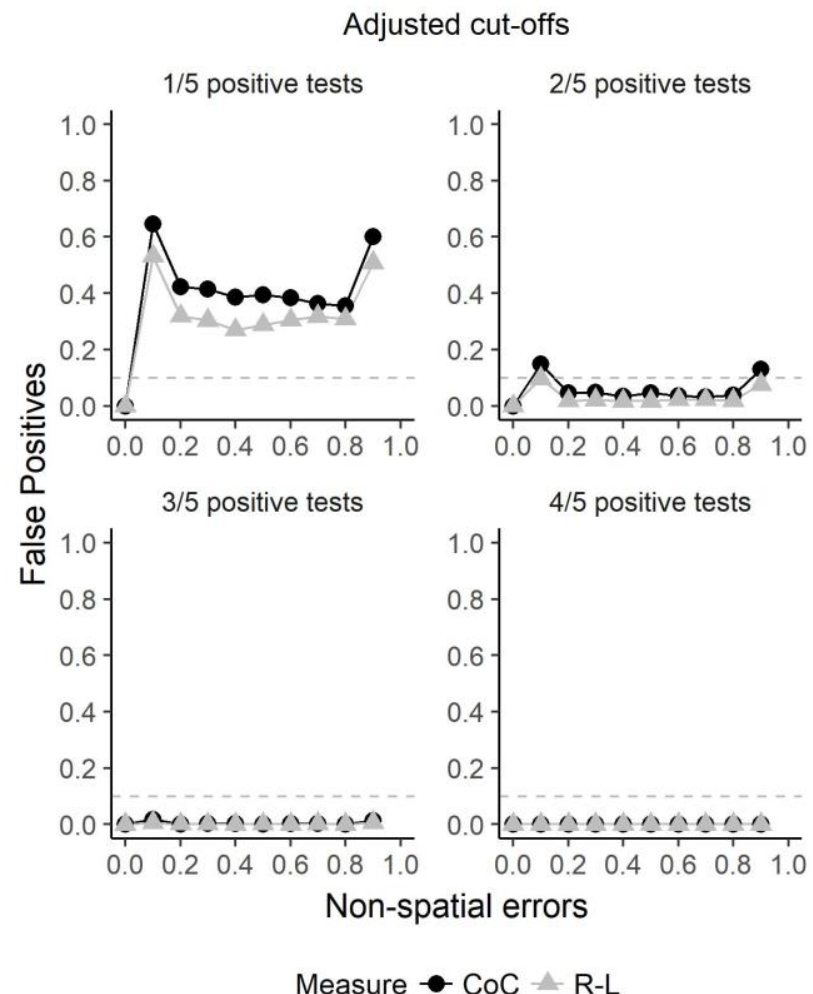

Figure 2. 
Huygelier-Spatial Neglect Diagnosis
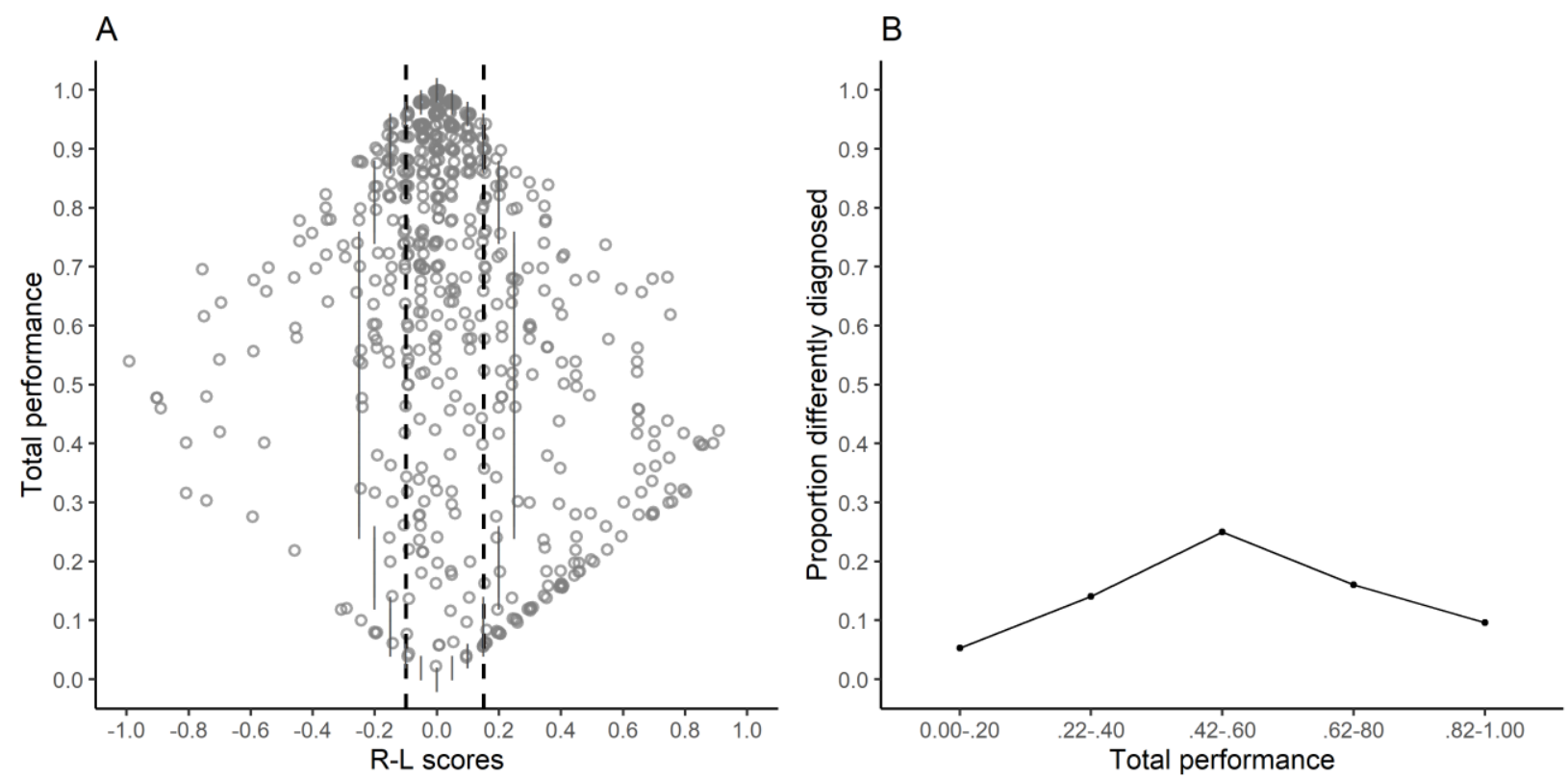

Figure 3. 